\title{
CARACTERIZAÇÃO E DINÂMICA DE COLONIZAÇÃO DE Cladosporium cladosporioides (Fresen.) DE VRIES EM FRUTOS DO CAFEEIRO (Coffea arabica L.)
}

\author{
Characterization and dynamic of colonization of Cladosporium cladosporioides (Fresen.) \\ de Vries in coffee fruits (Coffea arabica $\mathbf{L}$.)
}

\author{
Ricardo Tadeu Galvão Pereira ${ }^{1}$, Ludwig Heinrich Pfenning², Hilário Antônio de Castroº
}

\begin{abstract}
RESUMO
A presença de fungos em associação natural com frutos do cafeeiro é considerada um fator importante influenciando a qualidade do café. A influência negativa de algumas espécies de Aspergillus é conhecida, comprometendo inclusive a segurança do produto. Os relatos de fungos influenciando positivamente a qualidade se resumem à ocorrência de Cladosporium sp. associados a grãos que originaram cafés de boa qualidade, porém informações exatas sobre a espécie e a sua dinâmica no campo são escassas. Objetivando caracterizar a espécie associada ao cafeeiro e sua dinâmica de colonização, 18 isolados de Cladosporium foram caracterizados e identificados. A dinâmica de colonização do fungo nas comunidades externa e interna do fruto do cafeeiro foi estudada ao longo do período de desenvolvimento do fruto. A espécie associada ao cafeeiro foi identificada como Cladosporium cladosporioides (Fresen.) de Vries. A dinâmica do fungo é característica de um fungo saprófita encontrado em intensidade máxima quando os frutos estão nos estágios de cereja.
\end{abstract}

Termos para indexação: Café, qualidade, fungos.

\begin{abstract}
The natural occurrence of fungi in coffee fruits is considered an important factor influencing the quality of coffee. The negative effect of some Aspergillus species in coffee, which can also affect safety of the product, is well known. Otherwise, there are reports describing the positive influence of fungi in coffee quality, but they are limited to the occurrence of Cladosporium sp. in fruits, and its correlation with a product of good quality. Indeed, the exact information about the species involved and dynamics of colonization are not available. The objective of this work was to identify and characterize the species of Cladosporium, detected in coffee fruits, and study the dynamics of colonization of the fruits during the maturation process. The species found on coffee fruits was identified as Cladosporium cladosporioides (Fresen.) de Vries. and the dynamics of colonization showed the typical pattern of a saprophytic fungus, with its highest incidence when the fruits were in complete maturation stage.
\end{abstract}

Index Terms: coffee, quality, fungi.

(Recebido para publicação em 5 de março de 2004 e aprovado em 15 de fevereiro de 2005)

\section{INTRODUÇÃO}

A influência benéfica de fungos na qualidade de alguns alimentos é bem conhecida. Queijos, vinhos e salames possuem propriedades organolépticas que são conferidas pela ação de fungos específicos. Os primeiros relatos da influência de fungos sobre a qualidade do café datam de 1936, quando Krug (1940a) verificou, com auxílio de uma lupa de bolso em amostras de cafés ardidos, a presença de um fungo de micélio avermelhado identificado inicialmente como do gênero Fusarium. A partir daí, na série de trabalhos denominada "A origem dos cafés duros", o autor procura relacionar a má qualidade da bebida à presença de fungos, principalmente dos gêneros Fusarium, Aspergillus e Penicillium (KRUG, 1940a, b, c). Em vários estudos inferiu-se que a boa qualidade de café do tipo mole e dura tenha relação com a presença de Cladosporium nos frutos (ALVES \& CASTRO, 1998; CARVALHO et al., 1989; MEIRELLES, 1990). Em duas localidades do município de Lavras foi verificada a ocorrência de Colletotrichum sp., Phoma sp., Fusarium sp., Cladosporium sp. e Penicillium sp. em diferentes intensidades dependendo do estádio de desenvolvimento dos frutos do cafeeiro. Maior freqüência e intensidade de colonização por Cladosporium foram constatadas na área conhecida como produtora de café de boa qualidade (ALVES \& CASTRO, 1998).

Em outro estudo sobre a diversidade microbiana de cafés em diferentes estádios de maturação, realizado no região Sul de Minas Gerais, Cladosporium foi registrado com maior frequiência $(45,9 \%)$, seguido de Fusarium $(38,8 \%$ ),

\footnotetext{
'Engenheiro Agrônomo, Mestre em Fitopatologia - Universidade Federal de Lavras/UFLA - Cx. P. 3037 - 37.200-000 - Lavras, MG. ${ }^{2}$ Biólogo, PhD em Micologia - Professor Adjunto Departamento de Fitopatologia/UFLA.

${ }^{3}$ Engenheiro Agrônomo, Doutor em Fitopatologia - Professor Titular Departamento de Fitopatologia/UFLA.

*Parte da dissertação de mestrado do primeiro autor
} 
Aspergillus (2,2\%) e Penicillium (13,1\%), além dos genêros Monilia, Arthrobotrys e Beauveria em proporções menores (SILVA, 2000). O primeiro relato de Cladosporium em grãos de café foi feito por Bitancourt (1957), que observou Cladosporium com comportamento diferente dos outros fungos, não sendo encontrado no terreiro, mas no campo até o estágio de seco no pé. Entretanto, Alves \& Castro (1998) relataram a ocorrência de Cladosporium sp. em frutos nas fases, verde-cana, cereja, passa, seco no pé, grãos no chão e até no café beneficiado, com incidência maior na fase de passa e seco no pé.

Apesar da associação comum e ocorrência generalizada de Cladosporium em frutos do cafeeiro, a espécie ainda não foi caracterizada e identificada. $\mathrm{O}$ conhecimento da dinâmica de sua população em relação ao estágio de maturação do fruto pode resultar na proposição de práticas de manejo que favoreçam a melhoria da qualidade e a segurança do produto. Considerando a hipótese da associação de Cladosporium a grãos de café de boa qualidade, com o presente trabalho objetivou-se caracterizar e identificar Cladosporium associado aos frutos do cafeeiro e estudar a dinâmica de sua ocorrência no campo.

\section{MATERIAL E MÉTODOS}

\section{Caracterização e identificação de Cladosporium associado ao cafeeiro}

Foram coletados frutos, grãos, folhas e amostras de solo em lavouras nos locais relacionados na Tabela 1. Fragmentos de folhas, frutos e grãos foram transferidos para placas de Petri contendo meio MA2 (extrato de malte $2 \%+$ agar). As amostras de solo foram diluídas em série em água esterilizada e plaqueadas em meio MA2. A amostra de ar foi coletada abrindo-se uma placa contendo meio MA2 na lavoura por 15 minutos.
Após o período de incubação, esporos do fungo foram transferidos com uma agulha de vidro para placas de Petri contendo meio MA2 e incubados em temperatura ambiente. No meio MA2, foram observadas as características macroscópicas como cor e textura da colônia e taxa de crescimento. Posteriormente, os isolados foram transferidos para meio SNA $\left(\mathrm{KH}_{2} \mathrm{PO}_{4} 1 \mathrm{~g} ; \mathrm{KNO}_{3} 1 \mathrm{~g} ; \mathrm{MgSO}_{4}\right.$ 0,5 g; KCl 0,5 g; Glicose 0,2 g; Sacarose 0,2 g; Agar 20,0 g) e incubados a $25{ }^{\circ} \mathrm{C}$ por 10 dias. Blocos de $1 \times 1 \mathrm{~cm}$ colonizados pelo fungo foram diretamente retirados das placas e depositados sobre lâminas. Após aplicação de uma gota de água + glicerina (1:1), foram cobertos com lamínula. As análises de conidióforos e conídios foram feitas em microscópio óptico com objetiva de 40x. Foram obtidos e analisados 18 isolados de Cladosporium de diferentes substratos e locais (Tabela 1).

\section{Dinâmica de Cladosporium associado ao cafeeiro}

A dinâmica de colonização dos frutos por Cladosporium no campo foi avaliada mensalmente coletando-se uma amostra de sessenta frutos em uma lavoura localizada no município de Perdões - MG. Foram considerados dois ambientes distintos no fruto, a micobiota externa constituída pelos propágulos do fungo aderidos ao exocarpo superficialmente e a micobiota interna constituída pelos organismos presentes no mesocarpo e endosperma do fruto. A incidência do fungo na micobiota externa foi avaliada por meio de plaqueamento direto dos frutos em meio MA2. Para avaliar a incidência na micobiota interna, cortes longitudinais dos grãos foram depositados sobre meio MA2 em placas de Petri, após desinfecção dos frutos com $\mathrm{NaOCl} 5 \%$ por 5 minutos. A incidência de Cladosporium foi avaliada pela contagem do numero de grãos com colônias típicas do fungo e a identificação conforme metodologia descrita anteriormente.

TABELA 1 - Origem dos isolados de Cladosporium sp. utilizados na caracterização da espécie.

\begin{tabular}{clc|clc}
\hline Isolado & \multicolumn{1}{c}{ Local } & Substrato & Isolado & \multicolumn{1}{c}{ Local } & Substrato \\
\hline RT1 & Lavras, MG & Grão & RT10 & Lavras, MG & Fruto \\
RT2 & Lavras, MG & Grão & RT11 & Perdões, MG & Solo \\
RT3 & Lavras, MG & Ar & RT12 & Boa Esp., MG & Fruto \\
RT4 & Piumhi, MG & Folha & RT13 & Lavras, MG & Fruto \\
RT5 & Lavras, MG & Fruto & RT14 & Lavras, MG & Folha \\
RT6 & Lavras, MG & Fruto & RT15 & Perdões, MG & Folha \\
RT7 & Lavras, MG & Solo & RT16 & Lavras, MG & Fruto \\
RT8 & Perdões, MG & Folha & RT17 & Viçosa, MG & Fruto \\
RT9 & Lavras, MG & Solo & RT18 & Canaã, MG & Grão \\
\hline
\end{tabular}




\section{RESULTADOS E DISCUSSÃO}

\section{Caracterização e identificação de Cladosporium associado ao cafeeiro}

O conidióforo mede em torno de 100 (-245) x 2-5 m, é pouco pigmentado, liso ou pouco rugoso. O ramoconídio com 0 a 1 septo tem 2-5(-7) $\mathrm{mm}$ de largura e $15-32 \mathrm{~mm}$ de comprimento e apresenta cicatriz aparente. Os conídios medem 2-5 x 2-6 mm (maioria 2 x 4-6 mm), com formato elipsoidal a limoniforme, raramente subgloboso, de parede geralmente lisa, com pigmentação verde-oliva clara, formando longas cadeias (Tabela 2; Figura 1).

De acordo com as características descritas, a espécie é identificada como Cladosporium cladosporioides. As características distintivas que mais auxiliam na identificação dessa espécie são a cicatriz proeminente nos conídios, conidióforos sem nódulos, conídios sem septos ou com apenas um septo e, principalmente, o formato do conídio elipsoidal ou limoniforme.

TABELA 2 - Quadro comparativo das estruturas microscópicas dos isolados de Cladosporium cladosporioides analisados.

\begin{tabular}{cccc|cccc}
\hline Isolado & $\begin{array}{c}\text { Conídio } \\
\boldsymbol{\mu m}\end{array}$ & $\begin{array}{c}\text { Ramo- } \\
\text { Conídio } \\
\boldsymbol{\mu m}\end{array}$ & $\begin{array}{c}\text { Conídio- } \\
\text { foro } \boldsymbol{\mu \mathbf { m }}\end{array}$ & $\begin{array}{c}\text { Isolado } \\
\text { Conídio } \\
\boldsymbol{\mu m}\end{array}$ & $\begin{array}{c}\text { Ramo- } \\
\text { Conídio } \\
\boldsymbol{m}\end{array}$ & $\begin{array}{c}\text { Conídio- } \\
\text { foro } \boldsymbol{\mu \mathbf { m }}\end{array}$ \\
\hline RT1 & $7 \times 4$ & $15 \times 2$ & 88 & $\mathrm{RT} 10$ & $5 \times 2$ & $17 \times 2$ & 118 \\
RT2 & $6 \times 4$ & $29 \times 5$ & 245 & $\mathrm{RT11}$ & $7 \times 5$ & $32 \times 2$ & 110 \\
RT3 & $5 \times 3$ & $20 \times 5$ & 37 & RT12 & $6 \times 5$ & $15 \times 7$ & 135 \\
RT4 & $5 \times 4$ & $25 \times 5$ & 61 & RT13 & $5 \times 3$ & $24 \times 5$ & 147 \\
RT5 & $6 \times 5$ & $18 \times 4$ & 74 & RT14 & $5 \times 4$ & $29 \times 2$ & 98 \\
RT6 & $9 \times 5$ & $15 \times 2$ & 54 & RT15 & $6 \times 3$ & $24 \times 5$ & 100 \\
RT7 & $6 \times 4$ & $20 \times 5$ & 98 & RT16 & $5 \times 3$ & $27 \times 5$ & 86 \\
RT8 & $7 \times 3$ & $15 \times 5$ & 98 & RT17 & $7 \times 3$ & $20 \times 5$ & 90 \\
RT9 & $4 \times 2$ & $15 \times 2$ & 98 & RT18 & $5 \times 2$ & $17 \times 5$ & 76 \\
\hline
\end{tabular}

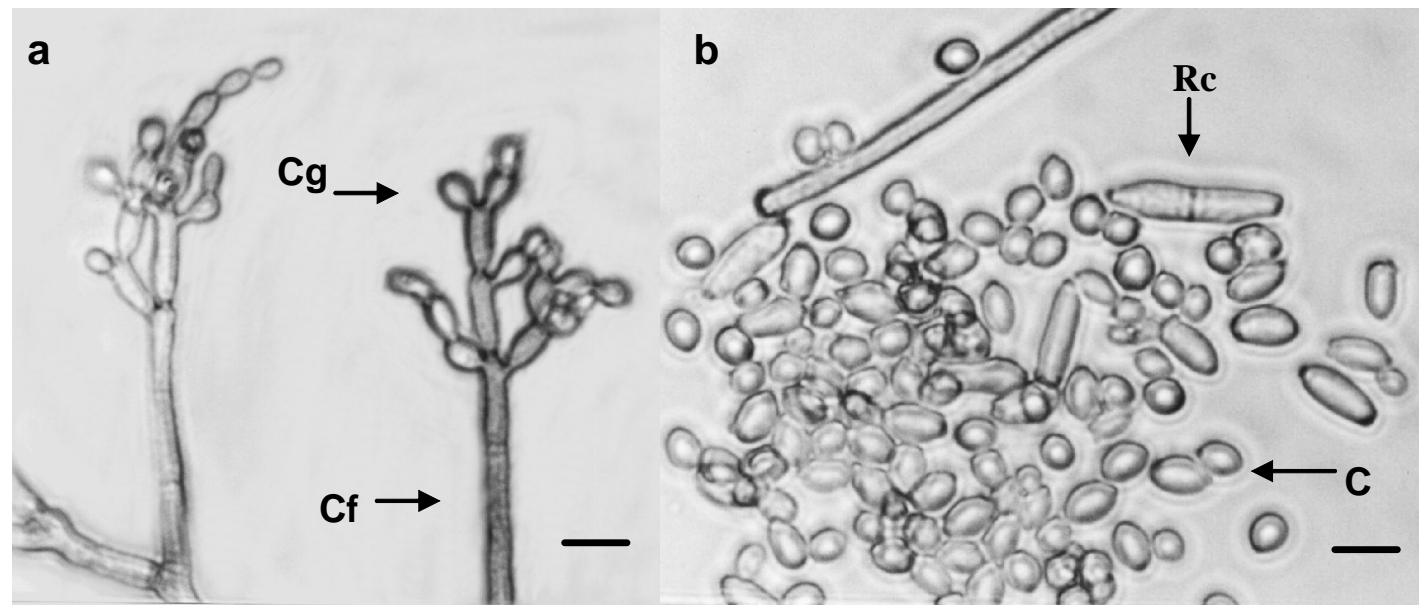

FIGURA 1 - Cladosporium cladosporioides a. Conidióforo (Cf) e célula conidiogênica (Cg); b. Ramoconídio (Rc) e conídio (C). Barra $=10 \mu \mathrm{m}$.

Ciênc. agrotec., Lavras, v. 29, n. 6, p. 1112 -1116, nov./dez., 2005 
Cladosporium cladosporioides é um dos fungos mais comuns, com ocorrência registrada em todas as partes do mundo. O fungo é encontrado como saprófita, contaminante do ar e alimentos, endofítico com função biológica importante na decomposição de matéria orgânica sendo também forte competidor com outros microrganismos (DOMSCH et al., 1993; ELLIS, 1971; SAMSON et al., 2000).

As colônias em MA2 crescem radialmente com coloração verde-oliva até marrom-esverdeado, textura aveludada, reverso em meio MA2 e DG18 preto. O tamanho das colônias após 10 dias a $25^{\circ} \mathrm{C}$ em MEA nunca ultrapassa $30 \mathrm{~mm}$. É facilmente isolado de solo, fruto, folhas e grãos do cafeeiro.

Cladosporium sphaerospermum Penz. é uma espécie bastante comum e semelhante a $C$. cladosporioides. A característica definitiva entre as duas é o formato do conídio que, nos isolados associados ao cafeeiro é característico, elipsoidal ou limoniforme, sendo raramente encontrado conídios com formato globoso ou subgloboso. Cladosporium cucumerinum Ellis e Arth. é também morfologicamente semelhante a $C$. cladosporioides, podendo ser diferenciado pela sua associação e patogênese a plantas da família das cucurbitáceas. Cladosporium tenuissimum Cooke apresenta similaridades com C. cladosporioides, mas seus conidióforos são mais longos com até $800 \mathrm{~m}$.

\section{Dinâmica de colonização de Cladosporium cladosporioides em frutos de café}

A dinâmica de Cladosporium cladosporioides é típica dos fungos saprófitas. Externamente, o fungo ocorre sobre o fruto durante todas as fases do desenvolvimento.
Inicialmente, nos meses de outubro até março, com os frutos nos estágios de chumbinho até verde-cana, esta colonização é menos intensa, não excedendo $25 \%$ dos frutos. Este fato se deve principalmente à presença de produtos fenólicos no fruto, como o tanino que apresenta ação fungitóxica (PIMENTA, 1995). A partir do mês de abril a colonização começa a se acentuar atingindo o índice máximo nos meses de maio e junho, quando o café já se encontra em estado de maturação avançado, chamado cereja (Figura 2). Estas observações estão de acordo com as de Alves \& Castro (1998), que relataram Cladosporium colonizando além das cerejas os frutos no estádio de passa. Internamente, o fungo começa a se manifestar a partir de março quando o fruto inicia sua maturação, a partir do mês de abril o fungo é encontrado facilmente na comunidade interna do grão. Os grãos utilizados para a análise não apresentavam qualquer sintoma de lesão provocada por fungos ou pragas, o que pode evidenciar o caráter endofítico de Cladosporium cladosporioides. O fungo foi encontrado em folhas de helicônias como endofítico, principalmente quando as folhas iniciam a senescência (PEREIRA, 1993). A mesma observação foi feita para folhas de cafeeiros sadios (CHAVES et al., 2002). A dificuldade de detecção de Cladosporium cladosporioides nas fases iniciais de desenvolvimento dos frutos pode estar relacionada ao impedimento de sua manifestação, pela inibição pelos próprios compostos do fruto, ou pela presença de outros como fungos como Phoma jolyana var. jolyana Pirozynki e Morgan-Jones que estava presente na maioria dos grãos em estádio verde cana. O aumento gradual do fungo coincide com a conversão dos compostos fenólicos em açucares facilitando a colonização interna do fruto.

\section{糃 Externa $\square$ Interna}

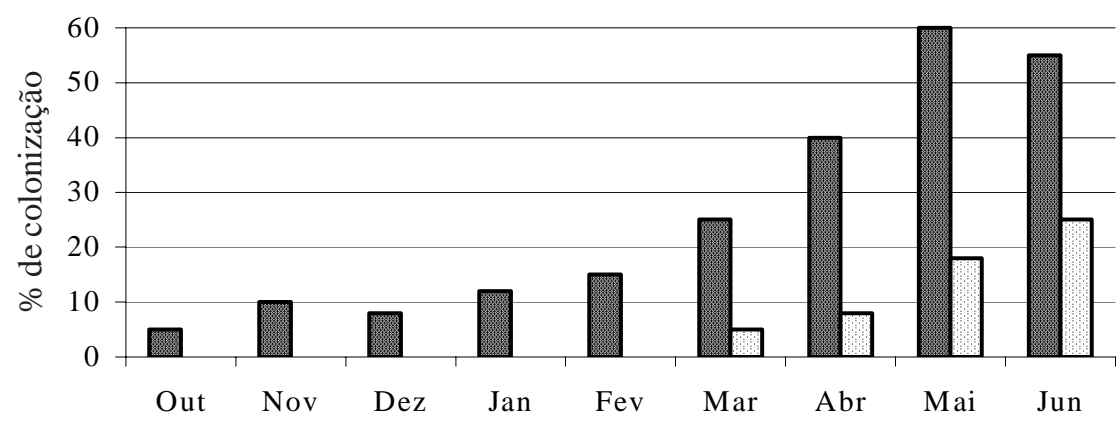

FIGURA 2 - Porcentagem de colonização por Cladosporium cladosporioides na comunidade interna e externa de frutos de café. 


\section{CONCLUSÕES}

Cladosporium cladosporioides é a espécie de Cladosporium associada ao fruto do cafeeiro. A população do fungo nos frutos aumenta na medida em que o fruto amadurece, atingindo seu ponto máximo no estádio cereja.

\section{AGRADECIMENTOS}

Agradecemos ao cafeicultor Dr. Odilon Teodoro Leite Filho e seu funcionário Sebastião, que gentilmente abriram as porteiras de sua propriedade para realização de parte deste trabalho.

\section{REFERÊNCIAS BIBLIOGRÁFICAS}

ALVES, E.; CASTRO, H. A. de. Fungos associados ao café (Coffea arabica L.) nas fases pré e pós-colheita em lavouras da região de Lavras. Summa Phytopatologica, Jaguariúna, v. 24, p. 4-7, 1998.

BITANCOURT, A. A. As ferramentas e podridões da cereja do café. Boletim da Superintendência dos Serviços do Café, São Paulo, v. 32, n. 359, p. 7-14, jan. 1957.

CARVALHO, V. D. de; CHALFOUN, S. M.; CHAGAS, S. J. de R. Relação entre classificação de café pela bebida e composição físico-química e química do grão beneficiado. In: CONGRESSO BRASILEIRO DE PESQUISAS CAFEEIRAS, 15., 1989, Maringá. Resumos... Rio de Janeiro: MIC/IBC, 1989. p. 25-26.

CHAVES, R. C.; PEREIRA, R. T. G.; CASTRO, H. A. Obtenção de isolados de Cladosporium com potencial de biocontrole pós-colheita. In: CONGRESSO DE INICIAÇÃO CIENTÍFICA DA UFLA, 15., 2002, Lavras. Anais... Lavras: UFLA, 2002. p. 191.

DOMSCH, K. H.; GAMS, W.; ANDERSON, T. H. Compendium of soil fungi. Federal Republic of Germany: IHV-Verlag, 1993. v. 1, 859 p.
ELLIS, M. B. Dematiaceous hyphomycetes. Wallingford: CAB International, 1971. 608 p.

KRUG, H. P. Cafés duros I. Revista do Instituto de Café do Estado de São Paulo, Campinas, v. 15, n. 159, maio 1940a.

KRUG, H. P. Cafés duros II. Revista do Instituto de Café do Estado de São Paulo, Campinas, v. 15, n. 163, p. 1393-1396, set. $1940 \mathrm{~b}$.

KRUG, H. P. Cafés duros III. Revista do Instituto de Café do Estado de São Paulo, Campinas, v. 15, n. 165, nov. 1940c.

MEIRELLES, A. M. A. Ocorrência e controle da microflora associada aos frutos de café (Coffea arabica L.) provenientes de diferentes localidades do estado de Minas Gerais. 1990. 71 f. Dissertação (Mestrado em Fitotecnia) Universidade Federal de Lavras, Lavras, 1990.

PEREIRA, J. A. Fungos endofíticos dos hospedeiros tropicais Stylosanthes guianensis e Musa cavendish. 1993. 135 f. Tese (Doutorado em Fitopatologia) - Escola Superior de Agricultura Luiz de Queiroz, Piracicaba, 1993.

PIMENTA, C. J. Qualidade do café (Coffea arabica $\mathbf{L}$.) originado de frutos colhidos em 4 estádios de maturação. 1995. 52 f. Dissertação (Mestrado em Ciência dos Alimentos) - Universidade Federal de Lavras, Lavras, 1995.

SAMSON, R. A.; HOEKSTRA, E. S.; FRISVAD, J. C.; FILTENBORG, O. Introduction to food and air-borne fungi. 6. ed. Baarn: CBS, 2000. 389 p.

SILVA, F. S. Diversidade microbiana em grãos de (Coffea arabica L.) processados por via seca nas pré e póscolheita. 2000. 105 p. Dissertação (Mestrado em Microbiologia dos Alimentos) - Universidade Federal de Lavras, Lavras, 2000. 